\title{
Principales creencias sexuales disfuncionales en mayores
}

\author{
MARTA BADENES-SASTRE \\ al225795@uji.es \\ Jesús Castro Calvo \\ castroj@psb.uji.es \\ RAFAEL BALLESTER-ARNAL \\ rballester@psb.uji.es
}

\section{Resumen}

Introducción: La sexualidad es una dimensión fundamental del ser humano e incluye el género, la identidad y la orientación sexual, el erotismo, la vinculación afectiva, el amor y la reproducción. Se manifiesta con pensamientos, fantasías, deseos, creencias, actitudes, valores, actividades prácticas, roles y relaciones y es el resultado de la interacción de múltiples factores. La respuesta sexual en mayores experimenta cambios con la edad. En este contexto, ciertas creencias disfuncionales sexuales pueden influir negativamente en el desarrollo de su sexualidad. Por ello nos planteamos conocer las principales creencias disfuncionales sexuales femeninas (CDSF) en personas mayores y comprobar si están relacionadas con la edad y el nivel educativo y socioeconómico. Método: Treinta mujeres de la Universitat per a Majors de la Universitat Jaume I de Castellón con edades entre 54 y 79 años (media $=61,43$; DT $=5,34$ ) cumplimentaron el cuestionario de creencias sexuales disfuncionales femeninas (CSDF, Nobre y PintoGouveia, 2002). Resultados: Las principales CSDF estuvieron relacionadas con el afecto y el amor en la pareja, los sentimientos maternales, la edad y el conservadurismo sexual. No se halló correlación significativa con la edad o el nivel educativo y socioeconómico a excepción de la edad con la dimensión de "Creencias sobre la autoimagen» $(r=0,483 ; p=0,007)$. Conclusión: Estos resultados indican que existen CSDF que pueden influir en la sexualidad de las personas. Así pues, con el objetivo de evitar la aparición de estas falsas creencias, prevenir trastornos sexuales y promocionar la salud sexual, la educación sexual sería relevante a lo largo de todo el ciclo vital.

Palabras clave: creencias sexuales disfuncionales femeninas, personas mayores, prevención trastornos sexuales y promoción salud sexual.

\section{Abstract}

Introduction: Hypersexual disorder is a clinical diagnosis characterised by an excessive sexual desire, together with a pathological inability to control it. It is estimated that this clinical diagnosis could affect about $6 \%$ of the total population, al- 
though this figure varies according to the instrument used for its diagnosis. One of the most important problems when accurately estimating its prevalence is related to the use of non-validated assessment instruments. In order to overcome this limitation, the preliminary results of the Spanish translation of the Hypersexuality Inventory $(\mathrm{IH})$ are presented herein. Method: For this purpose, 600 young adults (300 males and 300 females) aged 18-27 years old completed a translated and adapted version of the $\mathrm{IH}$, together with other instruments to assess sexual compulsivity. Results: By a factor analysis, we found that the $\mathrm{IH}$ comprised three subscales that explained $55.5 \%$ of variance. This factorial structure was confirmed through confirmatory factor analyses $\left(X^{2}=239.4 ;\right.$ G.L. $=145 ; X^{2} / G . L .=1.65 ;$ RMSEA $\left.=.03[90 \% \mathrm{Cl}=.02-.04]\right)$. Reliability for the total score and subscales ranged between .79 and .91 . The correlations between the $\mathrm{IH}$ and related measures were positive and significant ( $r$ between .74 and .71). Conclusion: This results support the employment of the IH to assess hypersexuality among Spanish young adults and its superiority to other traditional measures to assess its clinical diagnosis.

Keywords: Hypersexuality Inventory; Translation; Validation; Young adults

\section{Introducción}

La sexualidad es una dimensión fundamental del ser humano e incluye el género, la identidad y la orientación sexual, el erotismo, la vinculación afectiva, el amor y la reproducción. Así mismo, se manifiesta con pensamientos, fantasías, deseos, creencias, actitudes, valores, actividades prácticas, roles y relaciones. Así pues, la sexualidad es el resultado de la interacción de múltiples factores (Caballo, 2012). Es un área fundamental para la salud integral del individuo, siendo su estudio indispensable para el desarrollo de la salud mental de la población (Carreño, Guerra, Morales, Pimentel y Sánchez, 2002). A pesar de ello, la mayoría de personas crecen con conocimientos limitados en lo que refiere al ser humano sexuado, es decir, aspectos madurativos biológicos y emocionales relacionados con la propia sexualidad. Por ello es importante conocer los aspectos biológicos, psicosociales y conductuales en cada etapa de la sexualidad humana (Herrero, 2003).

Principalmente, los estudios se han centrado en la sexualidad de la población adolescente o adulta, siendo más escasos los centrados en personas mayores. Sin embargo, el envejecimiento va acompañado de características particulares que requieren un análisis más profundo (Cabello, 2012). Como ocurre en otras etapas, este proceso evolutivo puede acompañarse de creencias sexuales disfuncionales influenciadas por la cultura en la que existen mitos sexuales relacionados con el envejecimiento. Por ejemplo, pensar que las personas de edad no son sexualmente deseables, no tienen deseo sexual y no son sexualmente capaces (Hotvedt, 1983). Es cierto que, como indican Feldman, Goldstein y Hatzichristou (1994), la respuesta sexual podría verse modificada con la edad y que las disfunciones sexuales pueden repercutir en la calidad de vida. No obstante, aunque la persona pueda tener una respuesta excitatoria más lenta y un periodo refractario más largo, iguamente puede seguir experimentanto una sexualidad satisfactoria (Cabello, 2012).

Estudios realizados por Kaplan (1979), LoPiccolo y Friedman (1988) o Masters y Johnson (1970) explicaban desde un enfoque clínico y cualitativo la influencia que ejercen las creencias 
negativas y los mitos sexuales en el funcionamiento sexual. Más recientemente, Nobre y Pinto (2008) demostraron cómo las creencias disfuncionales ejercen mayor influencia en personas con disfunciones sexuales. Por otro lado, las creencias y actitudes sexuales positivas se relacionan con mayor satisfacción sexual (Truddel, 2002).

Algunas de las creencias culturales que encontramos sobre la sexualidad femenina son que el deseo sexual de las mujeres desaparece con la edad y que una vida sexualmente activa durante la vejez podría verse como inapropiada y reprobable. Otras ideas están relacionadas con la creencia de que la sexualidad tiene como fin el coito y la reproducción, que una vez tienen la menopausia se termina el deseo sexual y la feminidad, la exclusividad de las relaciones heterosexuales, el carácter pecaminoso del autoerotismo, la necesidad de estar enamorada para hacer el amor o que la mujer no debe tener la iniciativa en el sexo ni mostrar interés por el mismo, adoptando una postura pasiva (Freixas y Luque, 2009).

Por tanto, la evaluación y el estudio de las creencias sexuales en la población más adulta va a ser importante para determinar su influencia en la sexualidad humana y poder desarrollar programas de prevención y promoción de la salud sexual.

En este contexto, el objetivo del presente estudio es explorar las principales creencias sexuales disfuncionales en mujeres mayores y conocer si están relacionadas con la edad, el nivel socioeconómico y el nivel educativo de las participantes.

\section{Método}

\section{Participantes}

La muestra del estudio estuvo formada por 30 mujeres de la Universitat per a Majors de la Universitat Jaume I de Castellón con edades comprendidas entre 54 y 79 años (media = 61,43; DT $=5,34$ ). Del total de la muestra, el $10 \%$ eran solteras, un $50 \%$ estaban casadas o convivían con su pareja, un $9 \%$ estaban separadas o divorciadas y un $3 \%$ eran viudas. Con lo que respecta al nivel socio-económico de las participantes, el $20 \%$ presentaban un nivel socioeconómico bajo, un 76,7 \% medio y un 3,3\% alto. Por otro lado, un $30 \%$ poseía estudios primarios o graduado escolar, un 23,3\% tenía titulación FPI o FPII, un $30 \%$ eran diplomadas, un $13,3 \%$ licenciadas y un $3,3 \%$ tenía doctorado o máster.

\section{Instrumentos}

Para evaluar las creencias sexuales disfuncionales de las participantes se utilizó el cuestionario adaptado y validado por Ballester y cols. (en preparación) de la versión femenina del cuestionario Sexual Dysfunctional Beliefs Questionnaire (SDBQ) de Nobre y Pinto-Gouveia (2002). Consta de dos versiones, una para hombres y otra para mujeres. La versión femenina que nos compete muestra una fiablilidad test-retest de 0,8 y un alfa de Cronbach de 0,81. Esta formada por 40 ítems en los que la persona evaluada indica su grado de acuerdo/desacuerdo con diferentes afirmaciones según un tipo de respuesta de escala Likert que va de 1 (completamente en desacuerdo) a 5 (completamente de acuerdo). El análisis factorial del cuestionario muestra 6 dimensiones de creencias sexuales disfuncionales:

- D1. Conservadurismo sexual. Hace referencia a las creencias acerca del coito como aspecto central en la sexualidad humana. Algunas conductas sexuales como la masturbación, el sexo oral, etc. se entienden como algo desviado de la norma. Refleja la creen- 
cia del rol sexual de la mujer como algo pasivo y meramente receptivo interpretando la virginidad como algo muy valioso en las mujeres solteras. Está formada por los ítems 2 , $4,7,13,14,17,27,28$ y 32.

- D2. Deseo sexual y placer como pecado. Parte de la premisa de que el sexo es una actividad masculina donde la mujer debe controlar sus impulsos sexuales y placeres puesto que son experiencias pecaminosas. Está compuesta por los ítems 15, 34, 35, 36, 37 y 39.

- D3. Creencias sexuales relacionadas con la edad. Relacionan la disminución del deseo sexual, placer u orgasmo con el aumento de la edad, sobre todo en la postmenopausia. Consta de los ítems 5, 6, 8, 11 y 20.

- D4. Creencias relacionadas con la autoimagen. Considera la imagen corporal como un aspecto central en la sexualidad femenina. La conforman los ítems 10, 12, 38 y 40.

- D5. Primacía del afecto en las relaciones sexuales. El amor, el afecto y el acuerdo entre la pareja son los aspectos centrales en la sexualidad humana. La componen los ítems $1,3,18,22,23$ y 24.

- D6. Importancia de la maternidad. Explora el nivel de placer que las actividades maternales pueden suponer para la mujer, siendo la procreación la finalidad de cualquier experiencia sexual. Consta de los ítems $26,30,31$ y 33.

Por otro lado, los ítems 9 «Hay una variedad de maneras de conseguir placer y alcanzar el orgasmo», 16 «El orgasmo simultáneo entre los dos miembros de la pareja es necesario para que una relación sea sexualmente satisfactoria», 19 "Una carrera profesional brillante implica el control de las relaciones sexuales», 21 "Los hombres solo prestan atención a las mujeres jóvenes y atractivas», 25 "La mujer que inicia las relaciones sexuales es inmoral» y 29 «Si las mujeres se desinhiben sexualmente están totalmente bajo el control de los hombres» no se han incluido dentro de ninguna dimensión por razones estadísticas.

La puntuación total de cada dimensión se obtiene sumando la puntuación de cada ítem que la compone. A mayor puntuación, mayor cantidad de creencias sexuales disfuncionales tiene la persona. Los ítems 1, 3, 22, 23 y 24 han sido considerados inversos.

\section{Procedimiento}

En el marco de un estudio exploratorio para conocer las principales creencias sexuales disfuncionales entre la población, se administró el cuestionario adaptado de creencias sexuales disfuncionales femeninas (CSDF) de Nobre, Pinto-Gouveia y Gomes (2003) a treinta mujeres de la Universitat per a Majors de la Universitat Jaume I de Castellón que, una vez informadas sobre los fines de la investigación, lo cumplimentaron en formato de lápiz y papel y de manera voluntaria, individual y anónima.

\section{Análisis estadístico}

Los datos se analizaron por medio del paquete estadístico SPSS 22. Por un lado, se realizaron estadísticos descriptivos para conocer las principales creencias sexuales disfuncionales femeninas en la muestra. Por otro, se hicieron correlaciones bivariadas para conocer si existía relación entre la edad y el nivel educativo y socio-económico con las creencias sexuales disfuncionales femeninas. 


\section{Resultados}

Como vemos reflejado en la tabla 1, en la que se observan las en las que expresaban estar «De acuerdo» y "Totalmente de acuerdo», las creencias sexuales disfuncionales de mujeres mayores más frecuentes estuvieron relacionadas con «La finalidad del sexo para el hombre es estar satisfecho» y "Las emociones más maravillosas que puede experimentar una mujer son los sentimientos maternales». Con menor frecuencia (13,3\%) pero dentro de las principales CSDF encontramos las relacionadas con que «El sexo anal es una actividad perversa» o «Tras la menopausia las mujeres no consiguen llegar al orgasmo». Por otro lado, también aparecen con cierta prevalencia creencias como que «La masturbación es mala y dañina».

Tabla 1

Principales creencias sexuales disfuncionales

\begin{tabular}{lc}
\hline \multicolumn{1}{c}{ İ́tems } & Porcentaje acumulado \\
\hline $\begin{array}{lc}\text { 18. La finalidad del sexo para el hombre es estar satisfecho. } \\
\text { 31. Las emociones más maravillosas que una mujer puede experimentar }\end{array}$ & $46,7 \%$ \\
son los sentimientos maternales. & $30 \%$ \\
5. Después de la menopausia la mujer pierde su deseo sexual. & $26,7 \%$ \\
22. El sexo es una actividad bella y pura. & $26,7 \%$ \\
23. El sexo sin amor es como la comida sin sabor. & $26,7 \%$ \\
24. Todo está bien si ambos miembros de la pareja están de acuerdo. & $26,7 \%$ \\
2. La masturbación es mala y dañina. & $16,7 \%$ \\
1. El amor y el afecto de una pareja son necesarios para tener buen & $13,3 \%$ \\
sexo. & $13,3 \%$ \\
3. El componente más importante del sexo es el afecto mutuo. & $13,3 \%$ \\
8. Tras la menopausia las mujeres no consiguen llegar al orgasmo. & $13,3 \%$ \\
20. A medida que la mujer envejece, el placer que obtiene del sexo & $13,3 \%$ \\
decrece. & $13,3 \%$ \\
32. El sexo anal es una actividad perversa. & \\
33. En la habitación, la mujer es la jefa. &
\end{tabular}

En cuanto a las seis dimensiones que conforman las CSDF (véase la tabla 2), encontramos que el conservadurismo sexual y la primacía del afecto reportan el promedio más elevado, seguidas de creencias relacionadas con la edad y la concepción del placer y el deseo como pecado. Menor puntuación obtienen las creencias relacionadas con la imagen corporal o la maternidad. 
Tabla 2

Medias y desviaciones típicas de cada dimensión del cuestionario sobre csdf

\begin{tabular}{lcccc}
\hline \multicolumn{1}{c}{ Dimensión } & Media & DT & Rango & N \\
\hline $\begin{array}{l}\text { D1. } \\
\begin{array}{l}\text { Conservadurismo } \\
\text { sexual }\end{array}\end{array}$ & 15,17 & 5,05 & $9 / 29$ & 30 \\
$\begin{array}{l}\text { D2. Placer y deseo } \\
\text { sexual como } \\
\text { pecado }\end{array}$ & 8,30 & 2,72 & $6 / 14$ & 30 \\
$\begin{array}{l}\text { D3. Creencias } \\
\text { relacionadas } \\
\text { con la edad }\end{array}$ & 9,30 & 3,30 & & 30 \\
$\begin{array}{l}\text { D4. Creencias } \\
\text { relacionadas con la } \\
\text { imagen corporal }\end{array}$ & 5,97 & $5 / 16$ & 30 \\
$\begin{array}{l}\text { D5. Primacía } \\
\text { del afecto }\end{array}$ & 14,57 & 2,67 & $4 / 15$ & 30 \\
$\begin{array}{l}\text { D6. Primacía } \\
\text { de la maternidad }\end{array}$ & 7,90 & 3,59 & $6 / 21$ & 30 \\
\hline
\end{tabular}

Por otro lado, como podemos observar en la tabla 3, la única correlación significativa entre las dimensiones y las variables sociodemográficas se observa entre la edad y la dimensión 4 «Creencias relacionadas con la autoimagen», con una correlación de ,483 con una significación de, 007 .

Tabla 3

Correlaciones entre las dimensiones del sdbq y la edad y el nivel educativo y socioeconómico

\begin{tabular}{|c|c|c|c|c|c|c|c|}
\hline \multirow[b]{2}{*}{ Dimensión } & \multicolumn{2}{|c|}{ Edad } & \multicolumn{2}{|c|}{ NE } & \multicolumn{2}{|c|}{ NSE } & \multirow[b]{2}{*}{$\mathrm{N}$} \\
\hline & $r$ & $p$ & $r$ & $p$ & $r$ & $\mathrm{p}$ & \\
\hline $\begin{array}{l}\text { D1. } \\
\text { Conservadurismo } \\
\text { sexual }\end{array}$ & ,206 & ,265 &,- 042 & ,825 &,- 210 & ,265 & 30 \\
\hline $\begin{array}{l}\text { D2. Placer y deseo } \\
\text { sexual como pecado }\end{array}$ & , 133 & ,482 & ,069 & ,715 & ,069 & ,718 & 30 \\
\hline $\begin{array}{l}\text { D3. Creencias } \\
\text { relacionadas } \\
\text { con la edad }\end{array}$ & , 165 & ,385 &,- 030 & ,873 &,- 011 & ,953 & 30 \\
\hline $\begin{array}{l}\text { D4. Creencias } \\
\text { relacionadas con la } \\
\text { imagen corporal }\end{array}$ &, $483^{* *}$ & ,007 &,- 088 & 644 &,- 089 & ,641 & 30 \\
\hline
\end{tabular}




\begin{tabular}{lccccccc}
\hline & \multicolumn{2}{c}{ Edad } & \multicolumn{3}{c}{ NE } & \multicolumn{3}{c}{ NSE } \\
\hline \multicolumn{1}{c}{ Dimensión } & $\mathrm{r}$ & $\mathrm{p}$ & $\mathrm{r}$ & $\mathrm{p}$ & $\mathrm{r}$ & $\mathrm{p}$ & $\mathrm{N}$ \\
\hline $\begin{array}{l}\text { D5. Primacía } \\
\text { del afecto }\end{array}$ &,- 033 &, 862 &,- 256 &, 173 &, 017 &, 927 & 30 \\
$\begin{array}{l}\text { D6. Primacía } \\
\text { de la maternidad }\end{array}$ &, 249 &, 184 &, 111 &, 159 &, 045 &, 814 & 30 \\
\hline
\end{tabular}

\section{Discusión y conclusiones}

En base a los resultados obtenidos, podemos observar que una parte de las participantes presentan algún tipo de creencia sexual disfuncional. Entre las más frecuentes encontramos las relacionadas con la primacía del afecto entendida como la importancia del amor, el afecto y el acuerdo entre la pareja como algo central dentro de la sexualidad humana. Por ejemplo, dentro de esta dimensión son más comunes creencias como que el hombre tiene como finalidad en el sexo el estar satisfecho, que el sexo es algo bello y puro o que el amor y el afecto en la pareja son necesarios para tener buen sexo, lo que podria relacionarse con mitos existentes en estas mujeres mayores. Estas creencias pueden estar influenciadas por la cultura como indicaban Freixas y Luque (2009). Tanto la educación como las religiones han inculcado a las mujeres el concepto de sexualidad como algo relacionado con el amor, el afecto y la reproducción pero no con el placer y el disfrute. Esto puede ejercer su influencia en edades adultas, contribuyendo a una percepción insatisfactoria de la sexualidad.

Seguidamente, encontramos creencias relacionadas con la importancia de la maternidad, que se considera una de las experiencias emocionales más hermosas que se puedan experimentar, y con la edad en la sexualidad, donde las creencias disfuncionales sobre la menopausia hacen pensar que la mujer pierde el deseo sexual, disminuye el placer y ya no puede alcanzar el orgasmo. Coincidiendo con los mitos culturales que planteaban Freixas y Luque (2009), la concepción del sexo anal como algo perverso o la masturbación como algo malo y dañino está relacionada con creencias conservadoras que basan el sexo únicamente en el coito y donde la mujer adopta un rol pasivo.

También se ha observado una relación significativa positiva entre la edad de las participantes y la autoimagen corporal. Esta relación puede estar influenciada por la sociedad, pues en la vejez se producen cambios en la figura corporal más evidentes que pueden ser interpretados como amenazantes. Esto puede ser debido a que no encajan en el modelo impuesto por una sociedad de consumo donde prima el atractivo de una persona joven, con vitalidad y bella, tal y como señalaba Esguerra (2007).

Por otro lado, a pesar de que Gramegna y cols. (1998) y Laumann, Paik y Rosen (1999) encontraran que la educación es un factor de disminución del riesgo de trastornos sexuales, en nuestro estudio no encontramos significación en esta variable. Esto puede deberse a la evolución de la misma a lo largo del tiempo. De igual forma, consideramos importante diferenciar la educación general de la educación sexual para conocer su influencia en las creencias sexuales disfuncionales en la población.

Otros estudios como el realizado por Ruiz, Enrique y Ballester (2011) muestran cómo en personas jóvenes universitarias se hallan CSDF siendo las más comunes las relacionadas con la dimensión de primacía del afecto. Estos resultados coinciden con los obtenidos en el presente estudio. Así pues, la existencia de las creencias disfuncionales no es algo que únicamente se dé en población mayor. 
Al tener en cuenta estos resultados, cabe tener presente algunas limitaciones del estudio, como el tamaño de la muestra, que dificulta la generalización de los hallazgos a la población adulta general. De igual forma, el introducir un mayor número de variables psicosociales hubiera permitido profundizar sobre posibles factores moduladores de las creencias disfuncionales.

En cualquier caso, nuestro estudio pone en evidencia la existencia de creencias disfuncionales en población femenina adulta en relación, principalmente, con la primacía del afecto y el conservadurismo sexual. Seguidamente encontramos creencias relacionadas con la edad, el placer y el deseo como algo pecaminoso y la maternidad. También se halla una relación entre las creencias relacionadas con la imagen corporal y la edad.

A la luz de estos resultados, es necesario enfatizar en la prevención de futuras insatisfacciones sexuales y trastornos sexuales por medio de programas en los que se promocione la salud sexual y se trabajen las creencias sexuales disfuncionales de las personas adultas pues, a pesar de que el envejecimiento conlleva un deterioro, se puede seguir llevando una vida sexual activa y saludable.

\section{Referencias bibliográficas}

Blümel, J. E., Araya, M., Riquelme, R., Castro, G., Sánchez, F. y Gramegna, G. (2002). Prevalencia de los trastornos de la sexualidad en mujeres climatéricas. Influencia de la menopausia y de la terapia de reemplazo hormonal. Revista médica de Chile, 130, 11311138.

Cabello, F. (2010). Manual de sexología y terapia sexual. Madrid: Síntesis.

Carreño, J., Guerra, G., Morales, F., Pimentel, D. y Sánchez, C. (2002). Disfunción sexual masculina y calidad de la comunicación marital. Un estudio comparativo. Perinatología y Reproducción Humana, 16, 16-25.

Esguerra, I. (2007). Sexualidad después de los 60 años. Avances en Enfermería, 25 (2), 124140.

Freixas, A. y Luque, B. (2009). El secreto mejor guardado: La sexualidad de las mujeres mayores. Política y sociedad, 46, (2), 191-203.

Gramegna, G., Blümel, J., Roncagliolo, M., Aracena, B. y Tacla, X. (1998). Patrones de conducta sexual en mujeres chilenas. Revista Médica de Chile, 126, 162-168.

Herrera, A. (2003). Sexualidad en la vejez: ¿Mito o realidad? Revista chilena de obstetricia y ginecología, 68, 150-162.

Horveddt, M. (1983). The cross cultural and historical context. En R. B. Weg (ed.). Sexuality in the later year. New York: Academic.

Kaplan, H. (1979). Disorders of sexual desire and other new concepts and techniques in sex therapy. New York: Brunner Mazel.

Kaplan, H. (1979). Trastornos del Deseo Sexual. Barcelona: Grijalbo.

Laumann, E., Paik, A. y Rosen, R. (1999). Sexual Dysfunction in the United States. Prevalence and Predictors. Journal of the American Medical Association, 281, 537-544.

LoPiccolo, J. y Friedman, J. M. (1988). Broad spectrum treatment of low sexual desire: Integration of cognitive, behavioral and systemic therapy. En S. R. Leiblum y R. C. Rosen (eds.). Sexual Desire disorders (pp.107-144). New York: Guilford Press.

Masters, W. y Johnson, V. (1966). Respuesta sexual humana. Buenos Aires: Inter-Médica.

Masters, W. y Johnson, V. (1970). Human sexual inadequacy. Boston: Little, Brown \& Co.

Nobre, P. y Pinto-Gouveia, J. (2008). Cognitive and Emotional Predictors of Female Sexual Dysfunctions: Preliminary Findings. Journal of Sex \& Marital Therapy, 34, 325-342. 
Nobre, P., Pinto-Gouveia, J. y Gomes, F. (2003). Sexual Dysfunctionals Beliefs Questionnaire: an instrument to assess sexual dysfunctional beliefs as vulnerability factors to sexual problems. Sexual and Relationship Therapy, 18, 172-204.

Ruiz, E., Enrique, A. y Ballester, R. (2011). Creencias sexuales disfuncionales en población joven femenina: resultados preliminares. Fórum de recerca, 16, 1125-1140.

Trudel, G. (2002). Sexuality and marital life: Results of a survey. Journal of Sex \& Marital Therapy, 28, 224-229. 